\title{
Y. Allieu, J-L. Roux, G. Meyer Zu Reckendorf Arthropathies des métacarpo-phalangiennes et inter-phalangiennes de la main
}

\section{Sauramps Medical, 10-2008, 366 pp, broché, 67,00 €, ISBN 10: 2840235870, ISBN 13: 9782840235873}

\section{Pierre Kehr}

Received: 15 August 2009 / Accepted: 18 August 2009 / Published online: 1 September 2009

(C) Springer-Verlag 2009

Ce livre reprend les communications de la 2ème rencontre de l'institut montpelliérain de la main.

Il s'agit d'un livre multi auteurs sous la direction de Yves Allieu.

Il permet de faire le point sur les différents traitements des affections aussi bien arthrosique qu'inflammatoire comme la polyarthrite rhumatoïde.

Le livre est divisé en grandes parties parlant de l'IPD, de l'IPP, de la MCP, et de la colonne du pouce. Il permet de faire le point sur des sujets peu abordés comme la dénervation de l'IPP, la voie d'abord palmaire de l'IPP ou le traitement des lésions étagées de la colonne du pouce.
De nombreux chapitres sont consacrés aux prothèses IPP ou MP. Il s'agit d'une présentation des prothèses avec une série ou la technique opératoire. Le lecteur pourra se faire une idée des différentes indications mais aura du mal à retrouver un avantage de l'une sur l'autre.

Finalement un livre intéressant qui permet d'avoir une vision juste de l'état actuel des traitements des arthropathies des IPP et IPD.

No funds were received in support of this study.

Alain Graftiaux
P. Kehr ( $\square)$

Strasbourg, France

e-mail: kehrpier@aol.com 